\title{
Bulbar Myasthenia Gravis Superimposed in a Medullary Infarction Diagnosed by a Fiberoptic Endoscopic Evaluation of Swallowing With Simultaneous Tensilon Application
}

\author{
Sung-Jun Kim, MD, Geun-Young Park, MD, PhD, Yong-Min Choi, MD, \\ Dong-Gyun Sohn, MD, Sae-Rom Kang, MD, Sun Im, MD, PhD
}

Department of Rehabilitation Medicine, College of Medicine, The Catholic University of Korea, Seoul, Korea

\begin{abstract}
In the elderly, myasthenia gravis (MG) can present with bulbar symptoms, which can be clinically difficult to diagnose from other neurological comorbid conditions. We describe a case of a 75-year-old man who had been previously diagnosed with dysphagia associated with medullary infarction but exhibited aggravation of the dysphagia later on due to a superimposed development of bulbar MG. After recovering from his initial swallowing difficulties, the patient suddenly developed ptosis, drooling, and generalized weakness with aggravated dysphagia. Two follow-up brain magnetic resonance imaging (MRI) scans displayed no new brain lesions. Antibodies to acetylcholine receptor and muscle-specific kinase were negative. Subsequent electrodiagnosis with repetitive nerve stimulation tests revealed unremarkable findings. A diagnosis of bulbar MG could only be established after fiberoptic endoscopic evaluation of swallowing (FEES) with simultaneous Tensilon application. After application of intravenous pyridostigmine, significant improvement in dysphagia and ptosis were observed both clinically and according to the FEES.
\end{abstract}

Keywords Myasthenia gravis, Stroke, Edrophonium

\section{INTRODUCTION}

Myasthenia gravis (MG) is an autoimmune disorder caused by circulating acetylcholine receptor antibodies directed toward the postsynaptic neuromuscular membrane. Although MG is an uncommon disorder, it is being encountered increasingly in the elderly population. In particular, MG in the elderly may be confused with other

Received January 23, 2017; Accepted March 8, 2017

Corresponding author: Sun Im

Department of Rehabilitation Medicine, Bucheon St. Mary's Hospital, College of Medicine, The Catholic University of Korea, 327 Sosa-ro, Wonmi-gu, Bucheon 14647, Korea. Tel: +82-32-340-2182, Fax: +82-32-340-7640, E-mail: lafolia@catholic.ac.kr

ORCID: Sung-Jun Kim (http://orcid.org/0000-0002-4517-7294); Geun-Young Park (http://orcid.org/0000-0002-6587-6471); Yong-Min Choi (http:// orcid.org/0000-0001-7174-5489); Dong-Gyun Son (http://orcid.org/0000-0003-3274-9182); Sae-Rom Kang (http://orcid.org/0000-0002-7347-9169); Sun Im (http://orcid.org/0000-0001-8400-4911).

() This is an open-access article distributed under the terms of the Creative Commons Attribution Non-Commercial License (http://creativecommons.org/ licenses/by-nc/4.0) which permits unrestricted noncommercial use, distribution, and reproduction in any medium, provided the original work is properly cited. Copyright $\odot 2017$ by Korean Academy of Rehabilitation Medicine 
neurological disorders such as stroke, motor neuron disease, or Parkinson disease because the clinical features of MG resemble those other conditions. Consequently, physicians can misdiagnose MG in later life as a more common condition [1]. The diagnostic approach to MG is focused on confirming the clinical diagnosis. It generally presents with ptosis, diplopia, dysphagia, dysarthria, and the fatigable weakness of the bulbar and limbs muscles. Many testing tools including the Tensilon test, ice test, acetylcholine receptor antibody assays (anti-AChR Abs), repetitive nerve stimulation (RNS), and single fiber electromyography have been used in the diagnosis of MG [2]. A videofluoroscopic swallowing study (VFSS) and fiberoptic endoscopic evaluation of swallowing (FEES) can
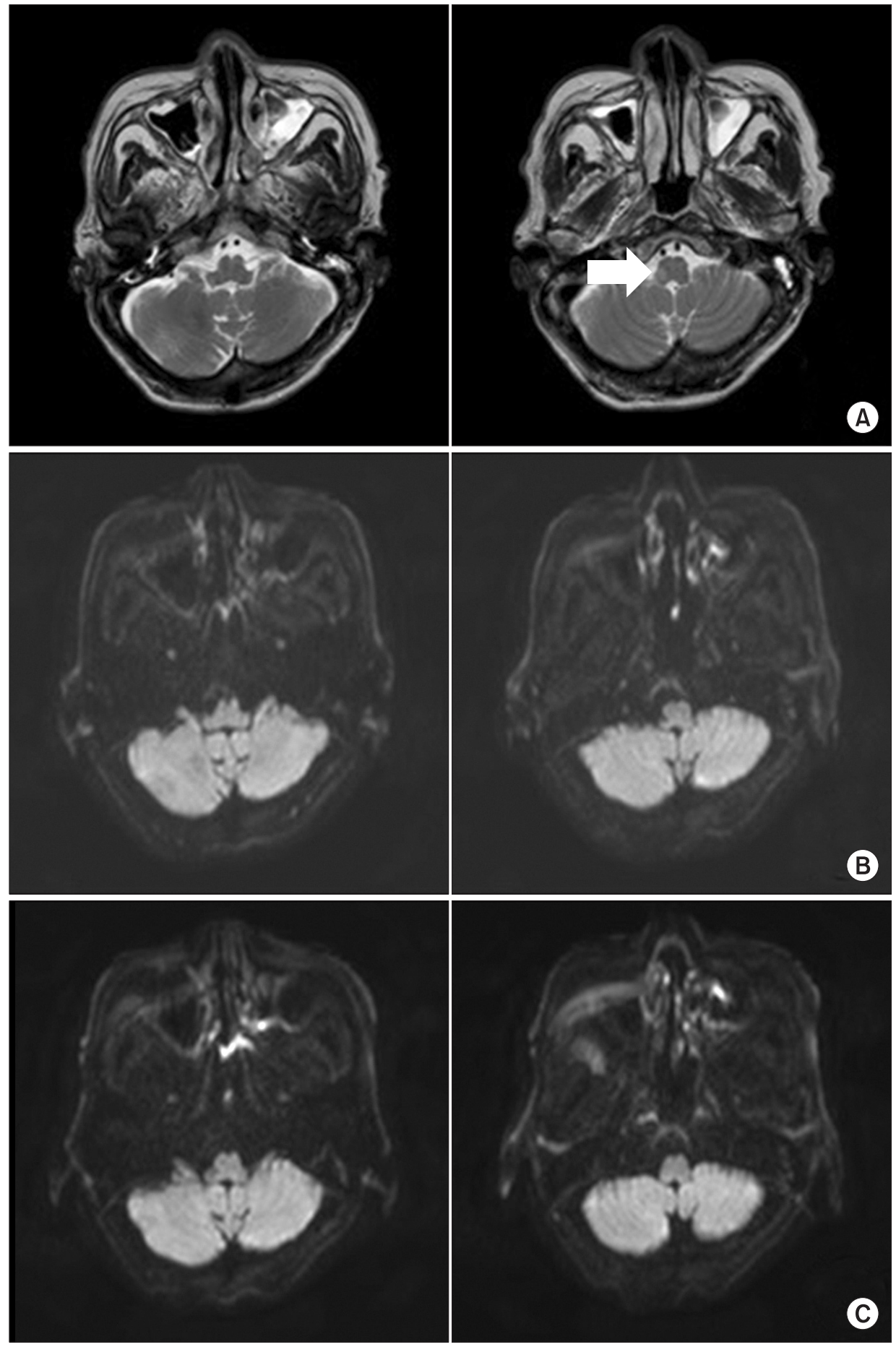

Fig. 1. (A) Initial brain magnetic resonance imagery (MRI) of T2weighted image show subacute infarction in the right medulla. After the onset of ptosis, a followup MRI of the brain demonstrated no acute lesion in the diffusion weighted image at (B) post 2 days and (C) 5 days. 
also assist in the diagnosis of MG-related dysphagia.

Recently, FEES with simultaneous Tensilon application (FEES-Tensilon test) was developed for establishing the diagnosis of MG-related dysphagia [3]. For the FEESTensilon test, FEES is performed with the simultaneous application of Tensilon to demonstrate reversibility of muscle weakness by blocking the action of an acetylcholinesterase, as in the Tensilon test. When significant functional improvement is demonstrated with the administration of Tensilon, a diagnosis of MG is made.

Both cerebral infarction and bulbar MG can cause dysphagia in the elderly. However, the presentation of these two disorders concomitantly in the same patient has rarely been reported. We report a case of a medullary infarction patient who suddenly developed ptosis, aggravated dysphagia, and dysarthria. A diagnosis of superimposed bulbar MG was made after performing the FEESTensilon test.

\section{CASE REPORT}

A 75-year-old man who had been diagnosed with dysphagia associated with medullary infarction 4 months prior (Fig. 1A) suddenly developed ptosis and increased drooling. A VFSS revealed dysphagia with reduced tongue movement in the oral phase, maximal amount or residue in the valleculae and pyriform sinus with overflow aspiration in the pharyngeal phase, and no compensation when a chin tuck was applied (Fig. 2A). The patient had previously undergone multidisciplinary rehabilitation including dysphagia rehabilitation. A prior VFSS (Fig. 2B) displayed reduced residues in the valleculae and pyriform sinus and mild aspiration only with liquid of up to level 5 on the Functional Oral Intake Scale (FOIS) (Table 1). He improved to a level that allowed him to be placed on a dysphagia diet with proper compensation and was discharged from the hospital.

Two weeks after discharge he was readmitted due to increased secretion and fever. A chest computed tomography revealed multifocal bronchopneumonia and he was treated with ampicillin-sulbactam. However, no aggravation of his swallowing function was observed and he was allowed to continue his oral feeding with a dysphagia diet and proper compensation. Due to the presence of a positive culture of extended-spectrum beta-lactamase from his secretion, his antibiotics were changed to meropenem. However, after 24 hours, he complained of ptosis; characterized by abnormal fatigability that improved with rest; and worsened slurred speech. Follow-up swallowing performance using the FEES revealed increased
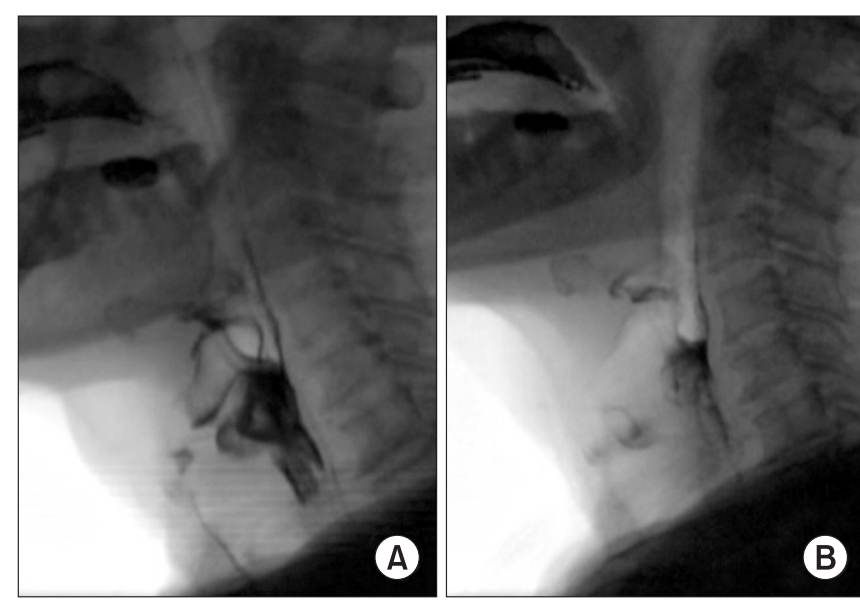

Fig. 2. (A) Six days after onset of ptosis, videofluoroscopic swallowing study (VFSS) images demonstrated maximal amount of residues in the valleculae and pyriform sinus with overflow aspiration in consistencies in the lateral view. (B) Five weeks prior to the onset of ptosis, VFSS images had initially revealed mild residues in the valleculae and pyriform sinus with only mild aspiration in the lateral view.

Table 1. Functional Oral Intake Scale (FOIS)

\begin{tabular}{cl}
\hline Level & \multicolumn{1}{c}{ Description } \\
\hline 1 & Nothing by mouth. \\
\hline 2 & Tube dependent with minimal attempts of food or liquid. \\
\hline 3 & Tube dependent with consistent oral intake of food or liquid. \\
\hline 4 & Total oral diet of a single consistency. \\
\hline 5 & Total oral diet with multiple consistencies, but requiring special preparation or compensations. \\
\hline 7 & Total oral diet with multiple consistencies without special preparation, but with specific food limitations. \\
\hline
\end{tabular}


saliva pooling with aspiration to the laryngeal vestibules. In comparison to the last study, his swallowing level had deteriorated from a FOIS level 5 to 1 . A recurrence of infarction was suspected; however, brain magnetic resonance imaging (MRI) performed at 2 and 5 days after the onset of ptosis showed no evidence of a recurrence of acute infarction (Fig. 1B, 1C). Nerve conduction studies revealed a normal range of latencies, amplitudes, and conduction velocities. A needle electromyography displayed no abnormal spontaneous activities at rest and a normal configuration of motor unit action potential. There was no definite electrodiagnostic evidence of peripheral neuropathy, motor neuron disease, or myopathy. A subsequent RNS was nonspecific with no decremental response recorded from the upper trapezius, biceps brachii, abductor pollicis brevis, orbicularis oculi, and masseter muscles.

Four days after the onset of ptosis, the patient had in- creased secretion and aggravation of his pneumonia. He complained of generalized fatigue; however, the medical research council scale for muscle strength revealed no paralysis of the extremity muscles. His antibiotics were changed to piperacillin-tazobactam.

Due to the technical challenges in making a proper diagnosis, he was referred to a neurologist and a Tensilon test was recommended. Due to the presence of aggravated swallowing performance, a FEES-Tensilon test was performed on the patient at 12 days after the onset of ptosis (Fig. 3). The test was performed according to protocol and recommendations [3]. The FEES displayed saliva aspiration continuous to the airway with a poor attempt to expectorate pooled secretions. After application of a cumulative dose of $5 \mathrm{mg}$ of intravenous pyridostigmine, the FEES showed improved swallowing performance with tolerable saliva swallowing, partial swallowing of boluses, and reduced residues in the valleculae and pyriform
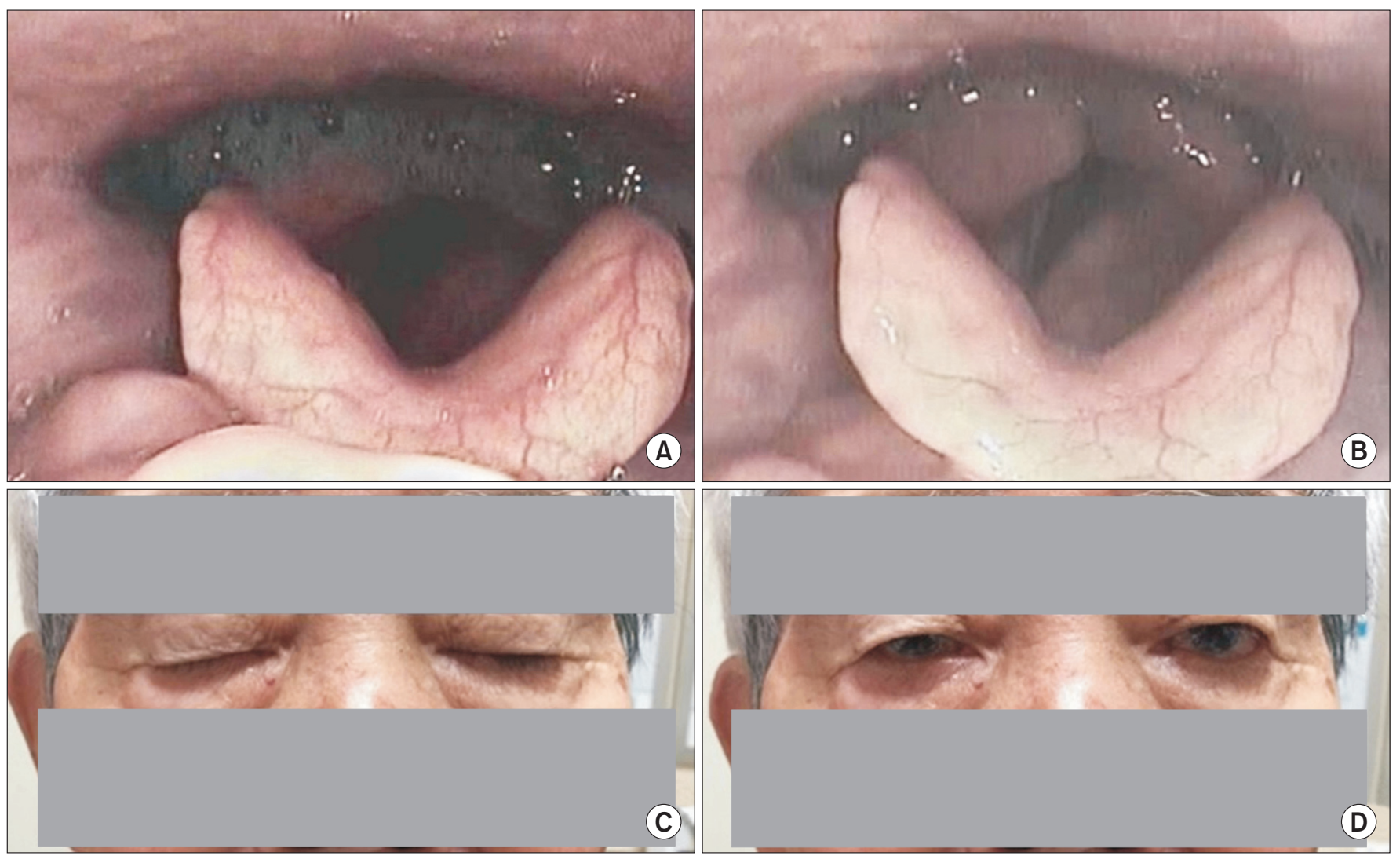

Fig. 3. Videoendoscopic findings of the FEES-Tensilon test displayed (A) at baseline, prior to Tensilon application, continuous silent saliva aspiration and pooling of secretions in the pyriform sinus and (B) after applying a cumulative dose of $5 \mathrm{mg}$ of Tensilon, improved clearance of secretions and tolerable swallowing of nectar bolus. (C) In comparison to pre-Tensilon application state, (D) ptosis significantly improved after applying a cumulative dose of 5 mg of Tensilon. FEES, fiberoptic endoscopic evaluation of swallowing. 
sinuses. His swallowing performance after Tensilon application improved with a FOIS level improvement from 1 to 3. The patient also showed resolution of diplopia. No complications such as bradycardia, respiratory distress, and hypotension were observed during the FEES-Tensilon test.

A dosage of $90 \mathrm{mg}$ pyridostigmine three times per day commenced. At 12 days post the onset of ptosis and after the resolution of pneumonia, $50 \mathrm{mg}$ of prednisolone once per day was initiated. The patient showed gradual improvement of ptosis and swallowing performance. The final results of the serum antibodies to anti-AChR and muscle-specific kinase (MuSK) titers were negative. At 6 months post onset of ptosis and swallowing deterioration, the patient's swallowing had recovered substantially, and his nasogastric tube was removed. He is now currently on regular diet.

\section{DISCUSSION}

MG is the most common disorder affecting neuromuscular transmission. However, in the elderly, it can be difficult to diagnose when it involves the bulbar muscles. In $15 \%$ of cases, bulbar symptoms may be the initial and sole presentation of MG [4]. It is often associated with seronegative antibodies [5], and the clinical challenges of performing direct RNS on the bulbar muscles can delay its proper diagnosis.

There have been several cases where MG was misdiagnosed as cerebral infarction $[6,7]$. All of the patients in the aforementioned cases received an initial impression of stroke or transient ischemic attack. However, the brain imaging scans displayed no evidence of acute infarction. MG was diagnosed later when symptoms deteriorated or distinctive symptoms characterized in MG, such as fatigue, were identified by obtaining a detailed and thorough patient history and conducting a physical examination. The diagnosis of these two separate disorders within the same patient has rarely been reported. According to the authors' knowledge, only one case of MG superimposed on a stroke patient has been reported previously [6]. Our case report is clinically relevant in that this rare presentation could be diagnosed with the application of a FEES-Tensilon test.

Some factors complicated the diagnosis of underlying MG in the present case. First, the patient had already swallowing disturbances when being diagnosed with medullary infarction. The deterioration of swallowing performance was therefore deemed to be associated with the recurring stroke rather than the development of another disorder. Second, serum antibody tests were negative in our patient, which are not detectable in approximately $15 \%$ of MG patients [2]. Furthermore, a few days are required to obtain the anti-AChR and anti-MuSK Abs results; therefore, other prompter and easier diagnostic studies are needed in the interim. Third, the negative RNS findings complicated the diagnosis. Although RNS is positive in $\mathbf{8 0 \%}$ of generalized MG, it is only positive in $50 \%$ of those with ocular presentations [2]. Considering that no generalized weakness was present in our patient and he showed only confined involvement of the ocular and bulbar muscles, it was highly likely that we would obtain a negative RNS in our patient. A positive decremental response is more likely when RNS is performed in a clinically weak muscle. However, in our patient, performing RNS directly on the bulbar muscles was not feasible. Fourth, before the onset of swallowing deterioration, the patient's ptosis was not remarkable. Especially in men, the eyelids may Droop down with increasing age. Aging causes a decrease of the total eyelid. Therefore, it is difficult to detect ptosis in the elderly [8]. It was only after the ptosis showed gradual deterioration with swallowing deterioration that combined oculobulbar MG was suspected.

MG may be exacerbated by a number of factors. Hospitalization, especially, can initiate various triggers of MG such as infection, emotional distress, surgery, and many classes of drugs. Many case reports have noted that MG was exacerbated by a number of antibiotics which impair neuromuscular transmission, including imipenem-cilastatin [9]. In our case, meropenem was one of the factors that may have triggered MG. It is important to precisely understand the provocative factors of MG and the contraindication to drug use.

The FEES-Tensilon test allows objective recording of swallowing performance improvement after Tensilon application with the FEES and has been suggested as a useful diagnostic tool in those with dysphagia due to unclear causes. Warnecke et al. [3] demonstrated many of its clinical advantages. First, it may be useful for isolated bulbar MG patients, especially when patients are in the seronegative MG as in our case. To the best of our knowledge, the 
current case study is one of the few reports that bulbar MG was diagnosed only through the FEES-Tensilon test. Second, it is useful in critically ill patients in the intensive care unit for a rapid diagnosis to confirm or exclude MG. Third, the test is helpful when distinguishing myasthenic and cholinergic crises. In addition to the above indications, it can be used to diagnose the rare presentation of superimposed development of bulbar MG as in our case. Finally, it is easy and safe to apply and can record the objective improvement of swallowing performance with the FEES after Tensilon application.

A VFSS with concomitant Tensilon administration can be performed to diagnose MG in patients with dysphagia [10]. Similar to the FEES-Tensilon test, when VFSS revealed improvement in dysphagia with Tensilon administration, MG can be diagnosed. However, the FEES-Tensilon test has advantages such as its ability to be performed at the bedside and without exposure to radiation [3].

In summary, this case report demonstrated the rare presentation of superimposed MG in a medullary infarction patient, which was successfully managed after proper diagnosis with the aid of an emerging novel test, the FEES-Tensilon test.

\section{CONFLICT OF INTEREST}

No potential conflict of interest relevant to this article was reported.

\section{REFERENCES}

1. Vincent A, Clover L, Buckley C, Grimley Evans J, Rothwell PM; UK Myasthenia Gravis Survey. Evidence of underdiagnosis of myasthenia gravis in older people. J Neurol Neurosurg Psychiatry 2003;74:1105-8.

2. Sathasivam S. Diagnosis and management of myasthenia gravis. Prog Neurol Psychiatry 2014;18:6-14.

3. Warnecke T, Teismann I, Zimmermann J, Oelenberg S, Ringelstein EB, Dziewas R. Fiberoptic endoscopic evaluation of swallowing with simultaneous Tensilon application in diagnosis and therapy of myasthenia gravis. J Neurol 2008;255:224-30.

4. Basiri K, Ansari B, Okhovat AA. Life-threatening misdiagnosis of bulbar onset myasthenia gravis as a motor neuron disease: How much can one rely on exaggerated deep tendon reflexes. Adv Biomed Res 2015;4:58.

5. Vincent A, McConville J, Farrugia ME, Newsom-Davis J. Seronegative myasthenia gravis. Semin Neurol 2004; 24:125-33.

6. Kleiner-Fisman G, Kott HS. Myasthenia gravis mimicking stroke in elderly patients. Mayo Clin Proc 1998; 73:1077-8.

7. Libman R, Benson R, Einberg K. Myasthenia mimicking vertebrobasilar stroke. J Neurol 2002;249:1512-4.

8. Aarli JA. Myasthenia gravis in the elderly: is it different? Ann N Y Acad Sci 2008;1132:238-43.

9. O'Riordan J, Javed M, Doherty C, Hutchinson M. Worsening of myasthenia gravis on treatment with imipenem/cilastatin. J Neurol Neurosurg Psychiatry 1994;57:383.

10. Schwartz DC, Waclawik AJ, Ringwala SN, Robbins J. Clinical utility of videofluorography with concomitant Tensilon administration in the diagnosis of bulbar myasthenia gravis. Dig Dis Sci 2005;50:858-61. 